BMJ Open

Sport \&

Exercise

Medicine

\title{
The effects of a single session of lumbar spinal manipulative therapy in terms of physical performance test symmetry in asymptomatic athletes: a single-blinded, randomised controlled study.
}

\author{
Bruno A P Alvarenga, ${ }^{1}$ Ricardo Fujikawa, ${ }^{2}$ Filipa João, ${ }^{1}$ Jerusa P R Lara, ${ }^{3}$ \\ António P Veloso ${ }^{1}$
}

To cite: Alvarenga BAP Fujikawa R, João F, et al. The effects of a single session of lumbar spinal manipulative therapy in terms of physical performance test symmetry in asymptomatic athletes: a single-blinded, randomised controlled study.. BMJ Open Sport \& Exercise Medicine 2018;4:e000389. doi:10.1136/ bmjsem-2018-000389

Accepted 3 September 2018

Check for updates

(C) Author(s) (or their employer(s)) 2018. Re-use permitted under CC BY-NC. No commercial re-use. See rights and permissions. Published by BMJ.

${ }^{1}$ Biomechanics and Functional Morphology Laboratory, Faculty of Human Kinetics, FMH, University of Lisbon, Lisbon, Portugal

${ }^{2}$ Department of Chiropractic, Real Centro Universitario Escorial - Maria Cristina, San Lorenzo de El Escorial, Spain ${ }^{3}$ Department of Physical Education, Federal University of Parana, Curitiba, Brazil

Correspondence to Bruno A P Alvarenga; brunofisioquiro@hotmail.com

\section{ABSTRACT}

Background and aim Musculoskeletal disorders in athletes, including spinal biomechanical dysfunctions, are believed to negatively influence symmetry. Spinal manipulative therapy (SMT) is recognised as a safe and effective treatment for musculoskeletal disorders, but there is little evidence about whether it can be beneficial in symmetry. Therefore, this study aimed to measure the effects of lumbar SMT in symmetry.

Methods Forty asymptomatic athletes participated in the study. The randomisation procedure was performed according to the following group allocation: group 1 (SMT) and group 2 (SHAM). Each participant completed a physical activity questionnaire, and also underwent clinical and physical evaluation for inclusion according to eligibility criteria. Statistical significance $(\mathrm{P}<0.05)$ between groups and types of therapy were calculated by physical performance tests symmetry (static position, squat and counter movement jump (CMJ), pre- and post-SMT and SHAM. There were 14 trials of three symmetry tests for each participant, for a total of 560 trials.

Results Lumbar SMT produced immediate effects in symmetry in the static position; however, the same effects were not found in squat and CMJ on symmetry 1 . Therefore, our results showed a significant difference in pre- (mean 16.3\%) and post-lumbar SMT (mean 3.7\%) in static symmetry. However, symmetry 2 showed no statistical significant differences for any of the tests and intervention groups. No statistically significant effects in symmetry pre- to post-SHAM were found in any of the tests.

Conclusions Statistically significant differences were found in lumbar SMT, but only for static symmetry. These findings suggest that SMT was effective in producing immediate effects in symmetry in the static position, but none in dynamic tests. Future studies could address our study's limitations.

Clinical trials register number NCT03361592.

\section{INTRODUCTION}

Musculoskeletal disorders in athletes, including spinal biomechanical

\section{What are the new findings}

- Asymptomatic athlete participants presented static bilateral asymmetry before the interventions, from pre- $16.3 \%$ and after interventions, reducing significantly to $3.7 \%$, immediately post-lumbar spinal manipulative therapy (SMT) intervention.

- The lumbar (SMT) intervention produced immediate effects on static symmetry, but the same effects were not found in dynamic tests (squat and counter movement jump).

- No statistically significant effects were found in symmetry, pre-to-post SHAM, in any of the physical performance tests.

How might it impact on clinical practice in the near future

In our randomised controlled study, lumbar SMT produced immediate effects on symmetry in the static standing position when applied therapeutically, but not in dynamic actions. Thus, this study expects to demonstrate that the single-session strategy of correcting the lumbar vertebral dysfunctions through lumbar SMT can effectively produce immediate effects in static symmetry.

- Adding new information regarding the static symmetry influenced by lumbar SMT intervention, these findings seem to be useful for clinical context in rehabilitation programmes of athletes.

dysfunctions, ${ }^{1-4}$ which are often asymptomatic, are believed to negatively influence physical performance in terms of symmetry. ${ }^{5-9}$

Asymmetric athletes may thus show decreased physical performance or have an increased risk for injury as a result of favouring the bilateral asymmetry of the body. ${ }^{1011}$ Tomkinson et al ${ }^{1012}$ suggested that 
athletes who are symmetric also have improved physical performance.

Bilateral asymmetry has been shown to be indicative of spinal abnormalities, ${ }^{513}$ and in clinical and sporting contexts, the ability to detect abnormal biomechanical parameters is extremely important when focusing on restoring normal function through the treatment strategies of these abnormalities. ${ }^{14-17}$ In this sense, we hypothesise that a therapeutic strategy for correcting spinal biomechanical dysfunctions through a lumbar spinal manipulative therapy intervention could produce immediate effects on symmetry.

Spinal Manipulative Therapy (SMT) is a safe and effective therapy for musculoskeletal disorders that has been increasingly utilised in sport. ${ }^{41-20}$ The purpose of SMT is to correct the biomechanical dysfunctions of spinal joints using a high-velocity low-amplitude movement, applied at the paraphysiological space, beyond the passive joint range of motion. ${ }^{1921-23}$

A recent systematic review of the literature ${ }^{18}$ showed several studies that associate SMT with sporting performance, but none of them has been focused on physical performance tests, namely in symmetry.

Nevertheless, several gaps in knowledge as well as a low level of evidence were found in the related scientific literature. ${ }^{351824}$ Therefore, to address these gaps, this randomised controlled study aimed to quantitatively measure the immediate effects of lumbar SMT on symmetry through physical performance tests: static standing position, squat movement, and counter movement jump (CMJ) in asymptomatic athletes.

\section{METHODS}

\section{Study design}

A single-blinded, single-session, randomised controlled study was conducted.

\section{Sample size calculations}

Based on prior sample size ${ }^{25}$ calculations, 40 athletes (20 females and 20 males) from different fields of sport participated in this study.

According to the relevant literature, this number of participants performing in multiple trials was sufficient and viable for application to this type of study, to ensure good statistical viability with regards to the parameters in question. $^{25} 26$

\section{Participants recruitment}

The participants were recruited through public advertisements at the Centre of High Performance, Faculty of Human Kinetics (FMH), University of Lisbon, Portugal, according to the triage process.

Ethical standards were applied according to the Helsinki Declarations, and the research protocol was approved by the Ethics Research Committee of the FMH University of Lisbon.

The CONSORT flow diagram, which is highly recommended for randomised clinical trials, ${ }^{27}$ was used as described in figure 1, which spans the time from enrolment, allocation and data collection procedures through the analysis of the data gathered from all the volunteer participants in this study.

\section{Eligibility criteria}

All selected participants were asked to fulfil the eligibility criteria.

Each participant underwent a clinical and physical evaluation, performed by an experienced physiotherapist and a chiropractitioner, to verify suitability for inclusion. The participants were athletes of any gender, aged between 18 and 35 years, and were asymptomatic and had a normal clinical evaluation.

The participants were physically active according to the "International Physical Activity Questionnaire" (IPAQ); short-form ${ }^{28}$ scores to standardise the sample including only active participants; medium-to-high level of physical practice, to ensure homogeneity.

Athletes who did not have the characteristics of an active person were excluded. Participants who experienced any changes in their training routine or competition during the study, and participants who had a history of spinal surgery and who were treated with manual therapy at any time during the study, were excluded. ${ }^{29}$

\section{Randomisation procedure}

After the eligibility criteria were fulfilled and the consent form was signed, participants were informed that the study protocol consisted of "therapeutic interventions" between physical tests.

The athlete participants were randomly divided into two groups by drawing from a black envelope containing the group assignment. All selected participants were asked to draw out one small ticket containing either the number 1 or 2 , referring respectively to group $1(n=20)$, who received the lumbar SMT intervention, and group 2 $(n=20)$, who received the SHAM intervention.

\section{Single-blinded: intervention mask procedure}

The intervention mask procedure was performed only for group assignment (SMT and SHAM interventions), thus establishing the single-blinded construct of the study. ${ }^{30}$ In fact, the participants were not made aware of whether a therapeutic intervention would reach a "mechanical effect" or whether it would have no effect, independent of the type of interventions. Although it is reasonable to suggest that participants may notice a physical difference after intervention, participants did not know to which intervention their group was allocated; participants only knew that they received one therapeutic intervention, as was initially explained.

\section{Biomechanical assessments}

The biomechanical model ${ }^{31-33}$ was created for use in static, dynamic and explosive actions. A total set of 49 reflective markers and five clusters were used during the data collection based on the calibrated anatomical system technique (CAST) ${ }^{32}$ 


\section{CONSORT 2010 Flow Diagram}

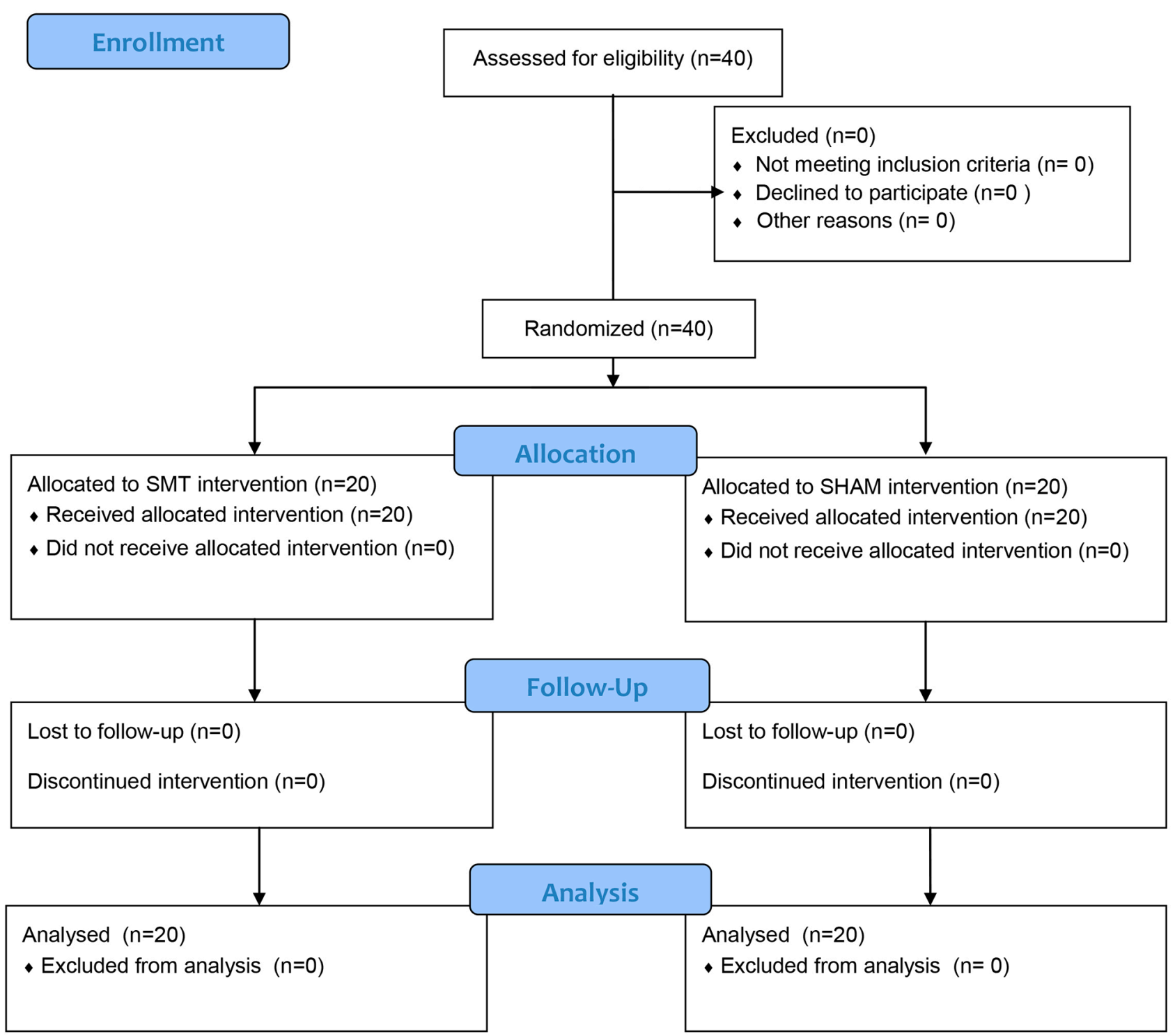

Figure 1 CONSORT flow-chart describing the randomised controlled study protocol. SMT, spinal manipulative therapy.

The motion capture system was equipped with an optoelectronic system of 15 cameras at $179 \mathrm{~Hz}$, and two force platforms (Kistler, Winterthur, Switzerland), operating at $500 \mathrm{~Hz}$, were utilised to collect the biomechanical parameters. ${ }^{34}$ Additionally, two symmetry indexes (\%) were used. The data were captured, processed and analysed using Qualisys QTM software (Gothenburg, Sweden) and Visual3D software (Version 5.01.18, C-Motion, Inc, Germantown, USA).

\section{Study protocol}

Physical performance tests symmetry (static position, free squat and CMJ) sequence, pre- and post-SMT and SHAM interventions, is presented in figure 2.
There were 14 trials of three physical performance tests symmetry (static position, free squat and CMJ) for each participant, for a total of 560 trials for all the athletes participating $(\mathrm{n}=40)$.

\section{Interventions}

The study interventions SMT and SHAM were performed by a chiropractitioner, as shown in figure $3 \mathrm{~A}, \mathrm{~B}$.

Spinal manipulative therapy

The lumbar SMT intervention was performed on the participants by a chiropractitioner using diversified techniques $^{35}$ that aim to correct the lumbar vertebral dysfunctional segments identified in the clinical assessments before the intervention. The participants were 


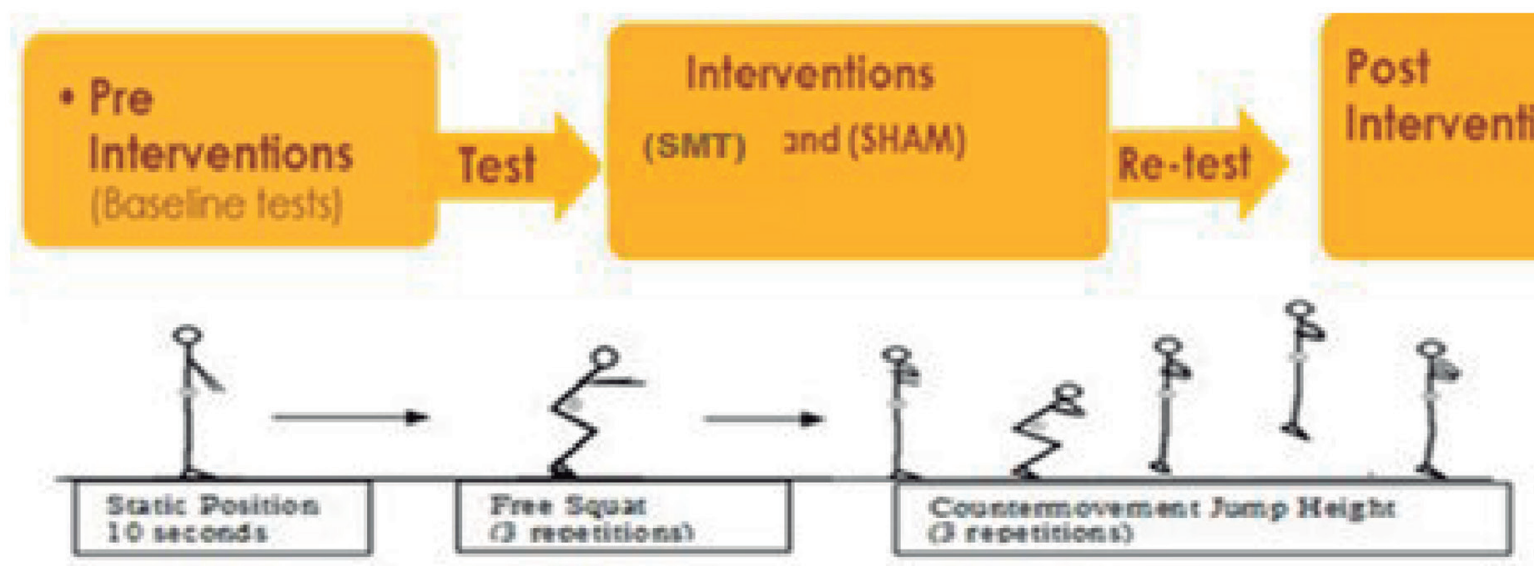

Phase PrezPefomance Tent - Befort SMTT Intervetion
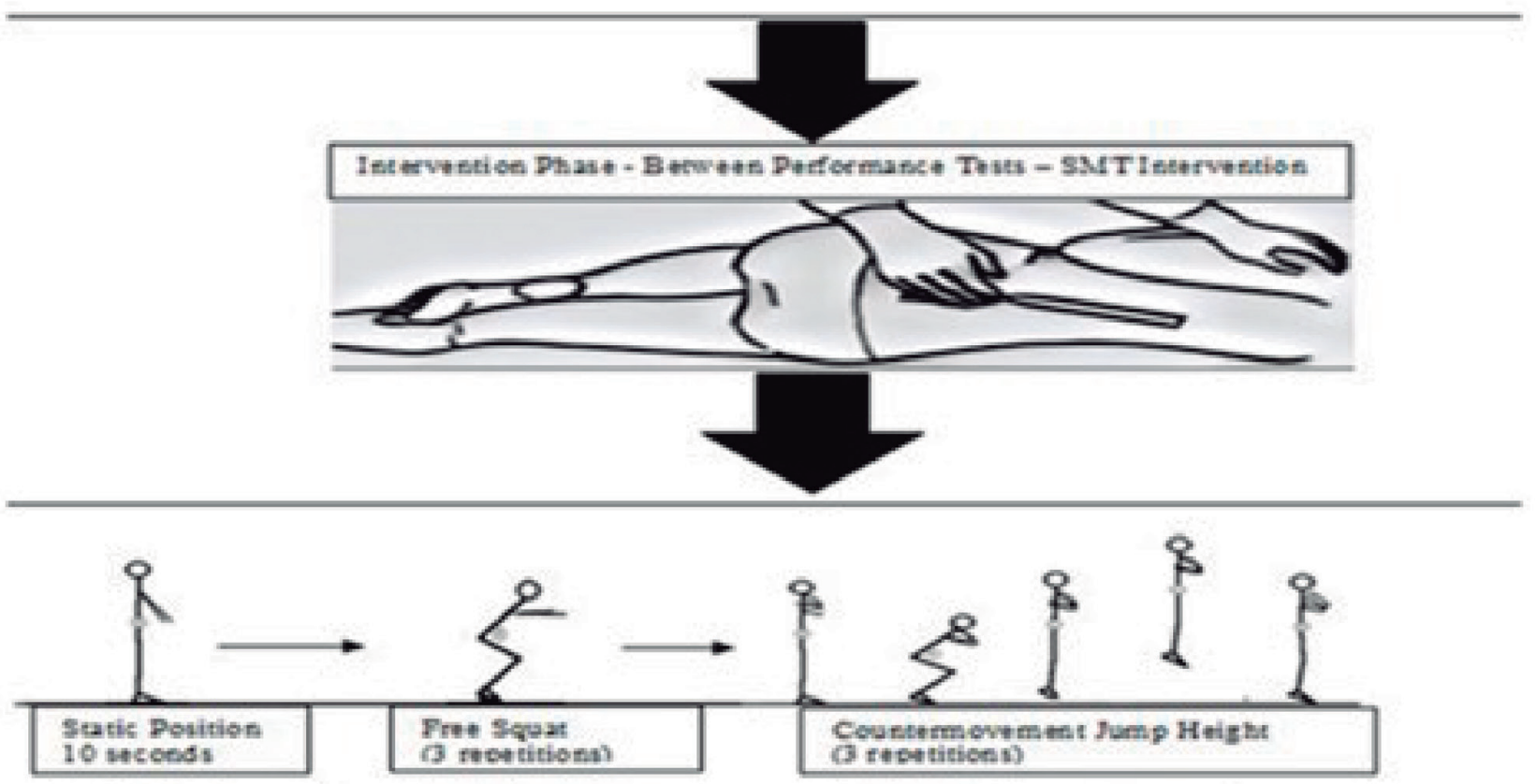

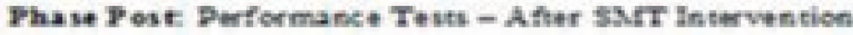

Figure 2 Study protocol, presenting physical performance tests symmetry (static position, free squat and countermovementjump) sequence, pre- and post-SMT and SHAM interventions.

instructed to lay down prone for the spinal motion palpation analysis, to evaluate the presence of dysfunction in the vertebral segments of the lumbar spine. The SMT was subsequently performed with the athlete laying sideways while a correction was performed contacting the lumbar, namely on the transverse process (mammillary) of the lumbar vertebrae, performing the lumbar roll technique as described by Liekens-Gillet and Bergmann ${ }^{35}$ (figure $3 \mathrm{~A}$ ).

SHAM control intervention

The SHAM procedure (pre-load SMT positioning) was performed with the participant in the lateral recumbent position, as described in the lumbar SMT intervention. The researcher guided the participant through the same motion as that in the SMT using the maintenance of the set-up position; however, no manipulative thrust was delivered. The chiropractitioner applied minimal pressure, and the position was maintained for approximately 1 min in total for both sides (figure $3 \mathrm{~B}$ ).

The SMT and SHAM interventions were both performed by a researcher with expertise in physiotherapy and chiropractice and more than 15 years of experience in clinical and sporting physical rehabilitation.

\section{Symmetry indexes}

Symmetry 1 (symmetry index, SI)

The SI index (\%) is the method most commonly used and cited in publication to report bilateral asymmetries in physical performance tests. ${ }^{7}$ 

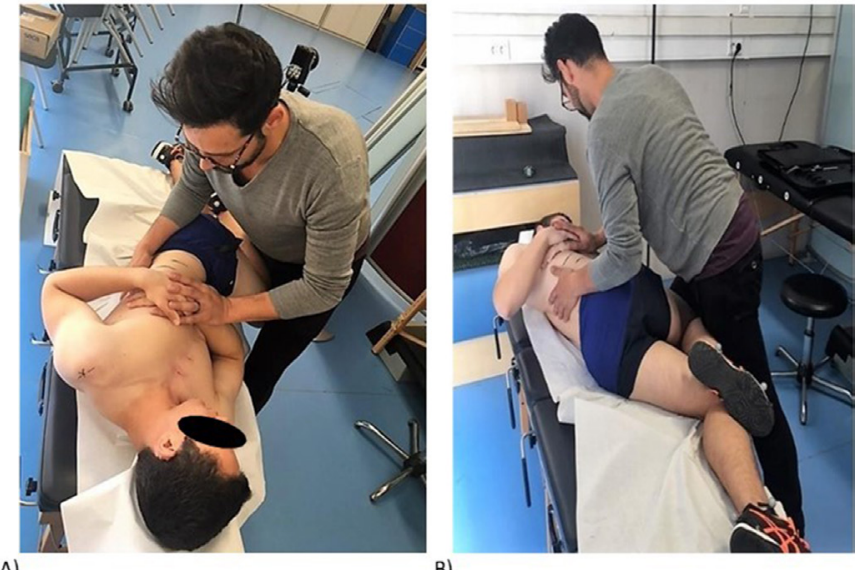

B)

Figure 3 (A) View of the participant receiving lumbar spinal manipulative therapy (SMT) intervention. (B) View of the participant receiving SHAM pre-positioning lumbar SMT intervention.

The symmetry measurement is the difference between two sides, known as SI, where $\mathrm{X}_{\mathrm{R}}$ is a measurement from the right side and $\mathrm{X}_{\mathrm{L}}$ is a homologous measurement from the left side (see equation below).

$$
\mathrm{SI}=\frac{\mathrm{XR}-\mathrm{XL}}{1 / 2(\mathrm{XR}+\mathrm{XL})} \times 100 \%
$$

The SI (\%), expressed as a percentage-with $0 \%$ representing perfect symmetry, indicating a more symmetrical pattern, and $100 \%$ representing complete asymmetry-was used to assess differences in the bilateral symmetry.

Symmetry 2 (linear global symmetry index, LGSI)

To calculate symmetry LGSI (\%) was used to measure the left and right sides in each performance test. Through this index, we were able to calculate the 3D components of the Euclidean distances from the "joint centres" to the pelvis origin, as illustrated in figure 4 .

The index was calculated as described by Cabral $e t a l,{ }^{36}$ adapted from the LGGA (linear global gait asymmetry) index, and is indicated in the following equation:

$$
L G S I=\sum_{v=v 1}^{v_{15}} \sqrt{\sum_{t=t_{1}}^{t_{101}}\left[x_{1}(t)-x_{r}(t)\right]^{2}}
$$

The 3D components of each vector are the input $\mathbf{v}$ in this index's equation, where $\mathbf{v}$ represents the angular variables (all three components of the hip, knee and ankle joint angles, the absolute pelvis angle, and the trunk angle in relation to the pelvis), and $\mathrm{xl}(\mathrm{t})$ and $\mathrm{xr}$ (t) are the values obtained for the left and right sides, respectively, at $\mathbf{t}$ (each percentage of the time-normalised performance test cycle). ${ }^{3637}$

\section{Statistical analysis}

Statistical calculations were performed using SPSS (version 24:IBM, IL), and Matlab software (MathWorks, Inc, USA).

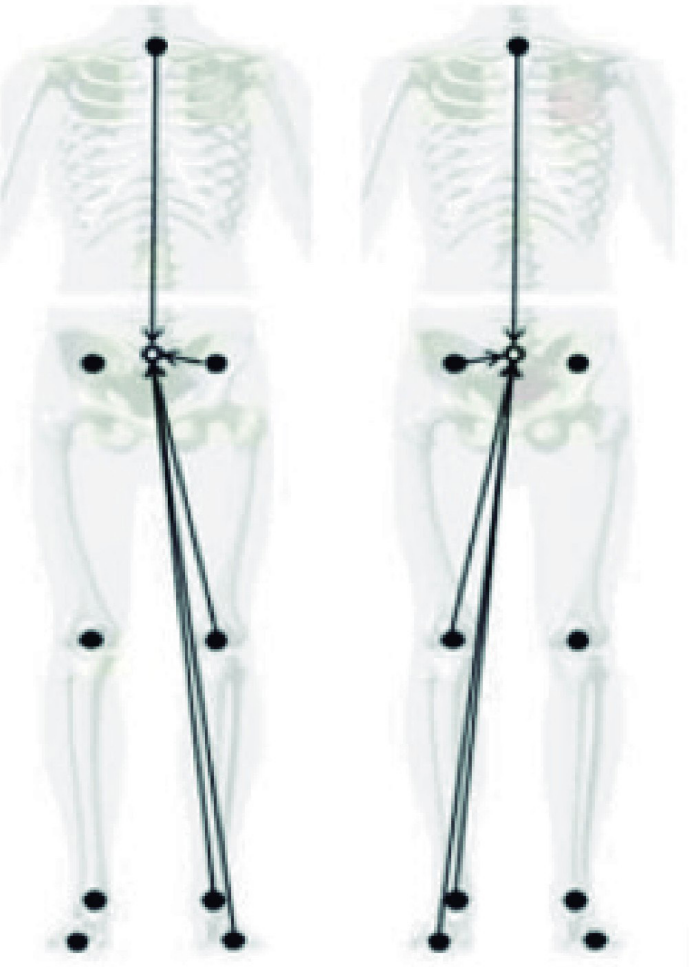

Figure 4 Vectors for the Euclidean distances computed during the right side and during the left side, for the linear global symmetry index (LGSI\%) calculations.

Kolmogorov-Smirnov tests were conducted before the statistical analysis, and they confirmed that the data were normally distributed. The significance of the differences between the means of the participant characteristic groups (age, weight and height) was determined by one-tailed, unpaired Student's t-test. The mean, SD and range (minimum and maximum) values of the selected variables were analysed. Differences between groups and pre- to post- instances of therapy were analysed using two-way analysis of variance (ANOVA). For all analyses $\mathrm{P}<0.05$ was considered statistically significant.

\section{RESULTS}

\section{Baseline participants' characteristics}

Based on the baseline participant characteristics, all participants were similar in regard to asymptomatic conditions, level of physical activity, and anthropometric characteristics.

The IPAQ classification values for all participants $(n=40)$ were calculated and demonstrated a high level of physical activity, with a mean score of $3.342 \mathrm{MET} / \mathrm{kg} /$ $\min$ and $\mathrm{SD}$ of $233 \mathrm{MET} / \mathrm{kg} / \mathrm{min}$.

Participants' anthropometric data were calculated and presented with a mean \pm SD for age of $23.8 \pm 5.3$ years, body mass of $63 \pm 7.5 \mathrm{~kg}$ and height of $1.68 \pm 0.06$ $\mathrm{m}$, respectively.

All participants completed the study and none of them reported any complaints during their participation. 


\section{Symmetry: outcome measures}

Group 1 (lumbar SMT)

Symmetry 1

Static standing position: The pre-phase (mean \pm SD) was $16.30 \pm 11.43 \%$, with a post-phase of $3.77 \pm 4.13 \%$. There were statistically significant differences in static symmetry ( $\mathrm{P}=0.01)$ immediately after lumbar SMT.

Free squat: The pre-phase was $9.37 \pm 6.9 \%$, with a post-phase of $10.27 \pm 7.70 \%$. There were no statistically significant differences.

CMJ : The pre-phase was $12.8 \pm 8.6 \%$, with a post-phase of $13.3 \pm 8.1 \%$. There were no statistically significant differences.

\section{Group 1 (lumbar SMT)}

Symmetry 2

Static standing position: The pre-phase was $1.48 \pm 0.48 \%$, with a post-phase measurement of $1.40 \pm 0.47 \%$. There were no statistically significant differences.

Free squat: The pre-phase was $1.86 \pm 0.51 \%$, with a post-phase of $1.82 \pm 0.61 \%$. There were no statistically significant differences.

CMJ: The pre-phase was $1.96 \pm 0.55 \%$, with a post-phase of $1.83 \pm 0.49 \%$. There were no statistically significant differences.

Group 2 (SHAM)

Symmetry 1

Static standing position: The pre-phase $($ mean \pm SD) was $10.75 \pm 10.50 \%$, with a post-phase of $9.02 \pm 6.18 \%$. There were no statistically significant differences.
Free squat: The pre-phase was $11.73 \pm 9.55 \%$, with a post-phase of $12.45 \pm 9.57 \%$. There were no statistically significant differences.

CMJ: The pre-phase was $13.99 \pm 8.76 \%$, with a post-phase of $12.40 \pm 8.59 \%$. There were no statistically significant differences.

\section{Group 2 (SHAM)}

\section{Symmetry 2}

Static standing position: The pre-phase was $1.30 \pm 0.40 \%$, with a post-phase of $1.46 \pm 0.52 \%$. There were no statistically significant differences.

Free squat: The pre-phase was $1.90 \pm 0.52 \%$, with a post-phase of $2.03 \pm 0.57 \%$. There were no statistically significant differences.

CMJ: The pre-phase was $2.04 \pm 0.66 \%$, with a post-phase of $1.99 \pm 0.49 \%$. There were no statistically significant differences.

The symmetry 1 and symmetry 2 outcome measures from both groups (SMT and SHAM) are visually presented by the box-plot diagram in figure 5 .

Statistically significance differences between pre- to post-SMT and between groups were found, and are presented in table $1 \mathrm{~A}$; table $1 \mathrm{~B}$ shows the range of symmetry values, pre- to post-SMT and SHAM interventions.

\section{DISCUSSION}

\section{Summary of main findings}

In our study, our participants presented bilateral asymmetry values initially in the static position, and post-SMT intervention these values reduced significantly.

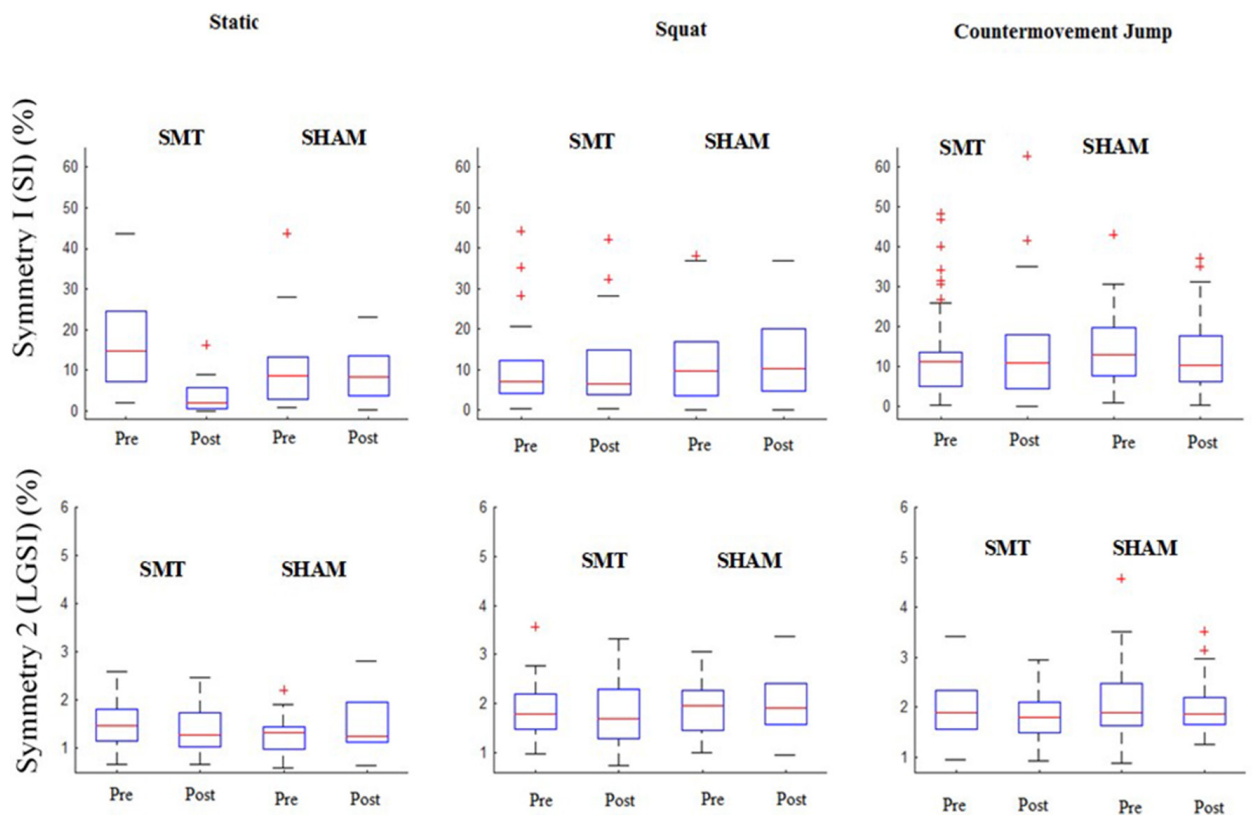

Figure 5 Visual representation of total symmetry values from both groups. Box-plot: the small black lines represent the interquartile, superior and inferior limit; the blue box represents the minimal and maximal values; the red line represents the groups means; and the red cross signals outliers, indicating variability. Both indexes (symmetry 1 and symmetry 2) show variability values of the physical performance tests (static, squat and counter movement jump). LGSI, linear global symmetry index; SI, symmetry index; SMT, spinal manipulative therapy. 
Table 1 (A) The mean (SD) values of two symmetry indexes (\%), Sym 1 and Sym 2, calculated for static trial (STT)free squat (SQT) and counter movement jump (CMJ), pre- and post- lumbar spinal manipulative therapy (SMT) and SHAM interventions for all participants. (B): The range (minimal and maximal) mean (M) values of two symmetry index (\%) were calculated for STT, SQT and CMJ pre- and post-, interventions, SMT and SHAM

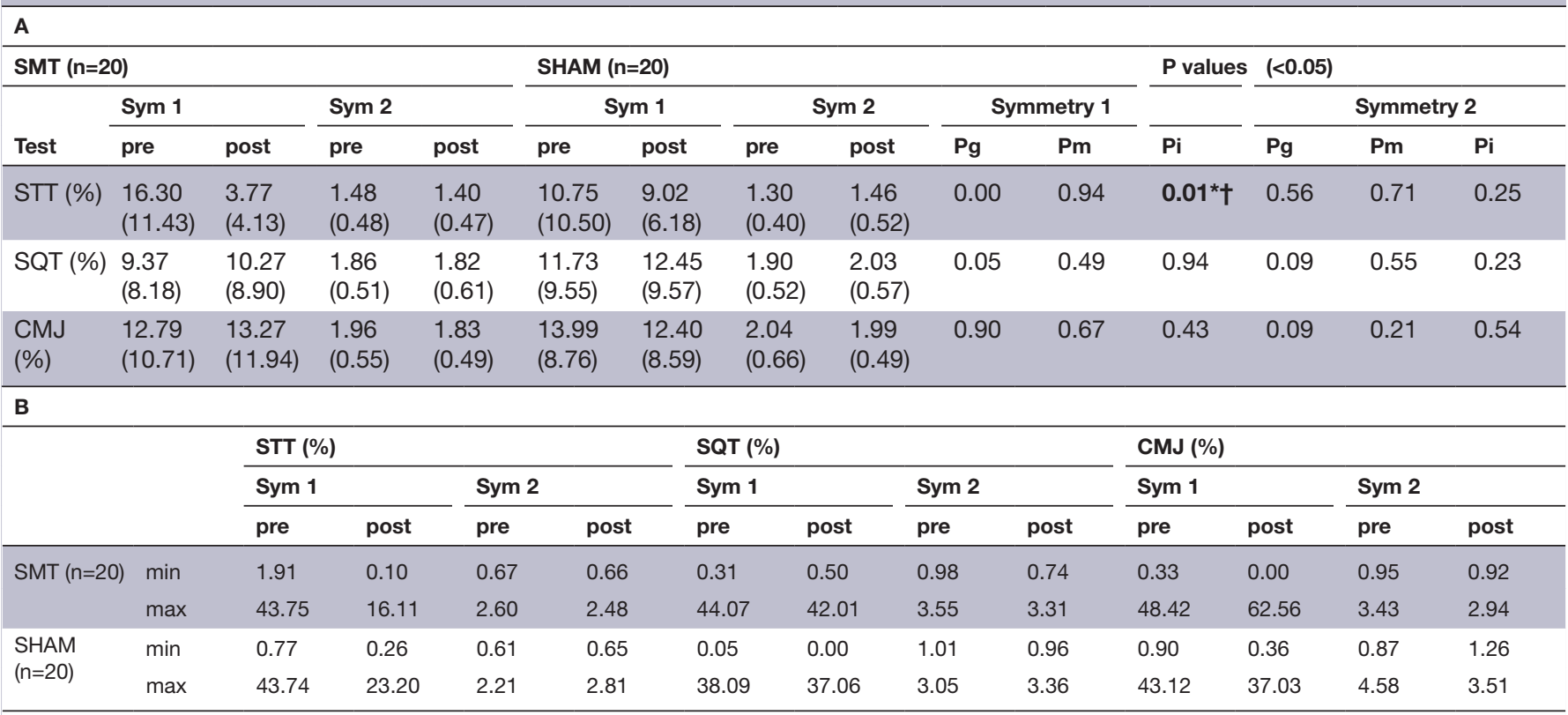

Significance difference between SMTpre $x$ SMTpost.

SMTpre $\times$ SHAMpost. $\mathrm{Pg}=\mathrm{P}$ value group: $\mathrm{Pm}=\mathrm{P}$ value moment; $\mathrm{P}=\mathrm{P}$ value interaction.

The lumbar (SMT) intervention produced immediate effects in static symmetry; however, the same effects were not found in the dynamic tests (squat and CMJ). Statistically significant differences were found between pre- and post-SMT intervention measurements, and between groups (SMT and SHAM), only for static symmetry. The symmetry 2 showed no statistical significant differences for any of tests, and in any of the groups.

The SHAM group showed no statistical significant differences between pre-and post-intervention measurements.

\section{Strengths and limitations}

Little evidence was found in the literature related to possible effects of SMT on symmetry in athletes. Surpassing this limitation, this study quantitatively measured physical performance test symmetry before and after lumbar SMT intervention to verify whether this intervention could effectively produce statistically significant effects.

The main limitation of this study relates to the blinding of the therapist to the intervention procedures performed on the participants. The double-blind procedure was not performed because it was incompatible with the protocol due to the inherent difficulty in blinding the therapist in this type of study. However, instrumental SMT, such as Activator, seems practical to perform these procedures and further investigations involving SHAM versus true interventions could consider incorporating the Activator instrument.
Another limitation was related to posture control variables that were not analysed because of the incompatibility of our protocol.

\section{Comparison and discussion of findings with respect to previous research}

Due to the little evidence found in the literature related to SMT on symmetry, our study was unable to compare results and discuss findings with other studies. Nevertheless, all our results in relation to those of other studies are discussed below.

Based on baseline group characteristics, both interventional groups of athletes were similar relative to asymmetry values, according to the literature. ${ }^{38-41}$

Before interventions, the participants presented asymmetry values in the SMT group with mean values of $16.3 \%$, and the SHAM group presented mean values of $10.7 \%$, indicating that some participants had considerable asymmetry.

Several authors who calculated symmetry based on performance tests $^{40} 42{ }^{43}$ determined the percentage of bilateral asymmetry with values of approximately $10-15 \%$. Differences $>15 \%$ are considered clinically significant. ${ }^{39-41}$

Despite some agreement in the literature regarding the asymmetry values of athletes in performance test-based assessments, Noyes $e t a l^{44}$ stated that a symmetry index of $85 \%$ or higher is acceptable as a normal range for both genders, and sport activity levels.

Nevertheless, our participants presented initially considerable bilateral asymmetry values in symmetry 1 
(table 1), and post-SMT intervention these values reduced significantly to what several authors, such as Herzog et $a l^{7}$ consider to be the minimum level of bilateral asymmetry, with asymmetry values ranging from $4-13 \%$.

\section{Clinical relevance and future directions}

By adding new information regarding the symmetry influenced by SMT intervention, this study expects to demonstrate that lumbar SMT can effectively produce immediate effects on symmetry in the static position, but not in dynamic actions, such as squat and CMJ. These findings seem to be useful in the clinical context of rehabilitative programmes for asymptomatic athletes.

Unfortunately, in order to be more relevant in terms of physical and sporting performance, our study would need to address other variables not found in this present study.

Future studies could be conducted, incorporating more variables, with short-term follow-up, and two or more groups crossed.

\section{CONCLUSIONS}

In our randomised controlled study, statistically significant differences were found between pre- and post-SMT, and between groups, for static symmetry only.

Lumbar SMT was shown to produce effects in bilateral symmetry in the static position when applied therapeutically. Therefore, our findings suggest that a single-session strategy of correcting lumbar vertebral dysfunction through SMT intervention was effective in producing immediate effects on symmetry in the static standing position. However, in dynamic tests (squat and $\mathrm{CMJ}$ ), pre- to post-lumbar SMT and SHAM were not statistically significantly different in terms of symmetry.

Future studies could address our study's limitations.

Acknowledgements The authors thank all the participants for their contributions to this study. The authors also thank the laboratory team for their assistance during the data collection and analysis process.

Contributors The randomised controlled study was completed, and all authors authorised the final version of the manuscript. The concept development; design; international clinical trial registration; local ethics council submissions; participant recruitment and allocation; clinical examinations; selection; experimental and control interventions, SMT and SHAM; data capture, collection and processing; biomechanics parameter and statistical calculations; analysis/interpretation; and writing of the report were performed by BAPA. The concept development, design, supervision, statistical analysis/interpretation and writing were performed by BAPA, RF, FJ, JPRL and APV. All other contributions to this work (critical review and writing a substantive part of the manuscript) were equally distributed among the authors.

Funding Doctorate Scholarship Funds to Bruno Alvarenga from Minister of Education Brazil - CAPES.

Competing interests None declared.

Patient consent Obtained.

Ethics approval FMH - Ethic Committee Register number: 31/2017.

Provenance and peer review Not commissioned; externally peer reviewed.

Data sharing statement All participants submitted a signed informed consent form of Faculty of Human Kinetics - FMH institutional consent that included information about the purpose of thestudy, its procedures, the participants' rights and welfare, participants' protections and the collection of data for publication. All data generated or analysed in this study were included in this material. The dataset used and/or analysed during the current study will be shared upon previous communication to the corresponding author, B.A.P.A, on reasonable request.

The datasets analysed are available from the Biomechanics and Functional Morphology Laboratory FMH, upon request and authorization. Faculty of Human Kinetics, University of Lisbon (Estrada da Costa, Dafundo. Lisbon, 1499-002, Portugal).

Open access This is an open access article distributed in accordance with the Creative Commons Attribution Non Commercial (CC BY-NC 4.0) license, which permits others to distribute, remix, adapt, build upon this work non-commercially, and license their derivative works on different terms, provided the original work is properly cited, appropriate credit is given, any changes made indicated, and the use is non-commercial. See: http://creativecommons.org/licenses/by-nc/4.0/

\section{REFERENCES}

1. Miners AL, Degraauw CA survey of Fellows in the College of Chiropractic Sports Sciences (Canada): their intervention practices and intended therapeutic outcomes when treating athletes. J Can Chiropr Assoc 2010;54:282-92.

2. Julian C, Hoskins W, Vitiello AL. Sports chiropractic management at the world ice hockey championships. Chiropr Osteopat 2010;18:32.

3. World Health Organization. WHO guidelines on basic training and safety in chiropractic. Geneva: World Health Organization, 2005.

4. Stump JL, Redwood D. The use and role of sport chiropractors in the national football league: a short report. J Manipulative Physiol Ther 2002;25:A2-5.

5. Guideline CP. Council on chiropractic practice guideline. Vertebral subluxation in chiropractic practice, 1998: 0-5.

6. Hannon SM. Objective physiologic changes and associated health benefits of chiropractic adjustments in asymptomatic subjects: a review of the literature. JVSR 2004;26:1-9.

7. Robinson RO, Herzog W, Nigg BM. Use of force platform variables to quantify the effects of chiropractic manipulation on gait symmetry. J Manipulative Physiol Ther 1987;10:172-6.

8. Herzog W, Nigg BM, Read LJ, et al. Asymmetries in ground reaction force patterns in normal human gait. Med Sci Sports Exerc 1989;21:110-4

9. Ga J, Herzog W, Kawchuk G. Biomechanical studies of spinal manipulative therapy (SMT): quantifying the movements of vertebral bodies during SMT. J Can Chiropr Assoc 1994;38:11-24.

10. Tomkinson GR, Popović N, Martin M. Bilateral symmetry and the competitive standard attained in elite and sub-elite sport. J Sports Sci 2003;21:201-11.

11. Bishop C, Turner A, Read P. Effects of inter-limb asymmetries on physical and sports performance: a systematic review. J Sports Sci 2018;36:1135-44.

12. Tomkinson GR, Olds TS. Physiological correlates of bilateral symmetry in humans. Int J Sports Med 2000;21:545-50.

13. Nigg BM, Herzog W. Biomechanics of the musculo-skeletal system. UK: Willey \& Sons, Chichester, 1994.

14. Pollard HWG, Pollard H, Ward G. Strength change of quadriceps femoris following a single manipulation of the L3/4 vertebral motion segment; a preliminary investigation. JNS 1996;4:137-44.

15. Grindstaff TL, Hertel J, Beazell JR, et al. Effects of lumbopelvic joint manipulation on quadriceps activation and strength in healthy individuals. Man Ther 2009;14:415-20.

16. Sports BiomechanicsBartlett R. Reducing injury and improving performance 2005:1-293.

17. Hoskins $W$, Pollard $H$. The effect of a sports chiropractic manual therapy intervention on the prevention of back pain, hamstring and lower limb injuries in semi-elite Australian Rules footballers: a randomized controlled trial. BMC Musculoskelet Disord 2010;11:64.

18. Botelho MB, Alvarenga BAP, Molina N. Spinal manipulative therapy and sports performance : a systematic review. J Manipulative Physiol Ther 2017;40:535-43.

19. Chapman-Smith D. Chiropractic: A profession in the field of health. Publisher Anhembi Morumbi, 2001.

20. Haldeman S. Spinal manipulative therapy in sports medicine. Clin Sports Med 1986;5:277-93.

21. Herzog W, Walter Herzog PD, et al. Clinical biomechanics of spinal manipulation. The mechanical, neuromuscular, and physiologic effects produced by spinal manipulation. Philadelphia: Churchill Livingstone, 2000: 206.

22. Pickar JG. Neurophysiological effects of spinal manipulation. Spine $J$ 2002;2:357-71.

23. Haldeman S. Neurological effects of the adjustment. J Manipulative Physiol Ther 2000;23:112-4.

24. Mansholt BA, Stites JS, Derby DC, et al. Essential literature for the chiropractic profession: a survey of chiropractic research leaders. Chiropr Man Therap 2013;21:33. 
25. Forrester SE. Selecting the number of trials in experimental biomechanics studies. International Biomechanics 2015;2:62-72.

26. Mullineaux DR, Bartlett RM, Bennett S. Research design and statistics in biomechanics and motor control. J Sports Sci 2001;19:739-60.

27. Eldridge SM, Chan CL. CONSORT 2010 statement: extension to randomised pilot and feasibility trials. Pilot Feasibility Stud. 2016, 2010.

28. International physical activity questionnaire (IPAQ). Guidelines for data processing and analysis- Short and Long Forms, 2005: 1-15.

29. Kankaanpää M, Taimela S, Laaksonen D, et al. Back and hip extensor fatigability in chronic low back pain patients and controls. Arch Phys Med Rehabil 1998;79:412-7.

30. Kawchuk GN, Haugen R, Fritz J. A true blind for subjects who receive spinal manipulation therapy. Arch Phys Med Rehabil 2009;90:366-8.

31. Lund ME, Andersen MS, de Zee M, et al. Scaling of musculoskeletal models from static and dynamic trials. International Biomechanics 2015;2:1-11

32. Cappozzo A, Catani F, Leardini A, et al. Position and orientation in space of bones during movement: experimental artefacts. Clin Biomech 1996;11:90-100

33. Seay J, Selbie WS, Hamill J. In vivo lumbo-sacral forces and moments during constant speed running at different stride lengths. J Sports Sci 2008;26:1519-29.

34. Antunes N, Medeiros FB, Alvares De CA. Analysis of the reliability of two force platforms for the simultaneous collection of kinetic variables. 33rd International Conference of Biomechanics in Sports 2015:1999-4168.
35. Bergmann TF PD, technique C. Principles and procedures. Philadelphia: Elsevier Mosby, 2010.

36. Cabral S, Fernandes R, Selbie WS, et al. Inter-session agreement and reliability of the Global Gait Asymmetry index in healthy adults. Gait Posture 2017;51:20-4.

37. Nigg S, Vienneau J, Maurer C, et al. Development of a symmetry index using discrete variables. Gait Posture 2013;38:115-9.

38. Almeida PDO, Prudente GFG, De Sá FE, et al. Postural and load distribution asymmetries in preschoolers. Motricidade 2016;11:58.

39. Menzel HJ, Chagas MH, Szmuchrowski LA, et al. Analysis of lower limb asymmetries by isokinetic and vertical jump tests in soccer players. J Strength Cond Res 2013;27:1370-7.

40. McGrath TM, Waddington G, Scarvell JM, et al. The effect of limb dominance on lower limb functional performance-a systematic review. J Sports Sci 2016;34:1-14.

41. Impellizzeri FM, Rampinini E, Maffiuletti $\mathrm{N}$, et al. A vertical jump force test for assessing bilateral strength asymmetry in athletes. Med Sci Sports Exerc 2007;39:2044-50.

42. Fousekis K, Tsepis E, Vagenas G. Lower limb strength in professional soccer players: profile, asymmetry, and training age. $J$ Sports Sci Med 2010;9:364-73.

43. Linthorne NP. Analysis of standing vertical jumps using a force platform. Am J Phys 2001;69:1198-204.

44. Noyes FR, Barber SD, Mangine RE. Abnormal lower limb symmetry determined by function hop tests after anterior cruciate ligament rupture. Am J Sports Med 1991;19:513-8. 